\title{
Electron spin relaxation of photochemically generated radical pairs diffusing in micellar supercages
}

\author{
U.E. Steiner and J.Q. Wu \\ Fakultat für Chome, Linverstat Konstanz, W'-7750 Konstanz, Germany.
}

\begin{abstract}
A kınetıc analysıs of previous experımental data [T. Ulrıch and U.E. Steiner, Chem. Phys. Letters 112 (1984) 365 ] on the magnetic-field dependent recombination kıtuetics of radical pairs photochemically produced with triplet spin corrclation in waterin-oil microemulsion droplets of variable size $\left(r_{\mathrm{M}}\right)$ provided the $r_{\mathrm{M}^{-}}$and field-dependence of the rate constant $k_{\mathrm{r}}$ of the $\mathrm{T}_{ \pm} \overrightarrow{\mathrm{F}}, \mathrm{T}_{\mathrm{0}}$ spin relaxation process. It was found independent of $r_{\mathrm{M}}$ at low fields, but strongly decreasing with increasing $r_{\mathrm{M}}$ at fields $\gtrsim 100 \mathrm{G}$. The latter behaviour was attributed to the mechanısm of electron-spin dipolar interaction. Quantitative treatments of this relaxation mechanısm are provided ( 1 ) by adaptıng Torrey's analytical result for homogeneous solution to the situation of one radical pair in a micellar supercage and (11) by Monte Carlo calculation of the autocorrelation function of the pertinent perturbation matrix element, considering diffusion in the volume or on the surface of the supercage. The electron-spin dipolar relaxation mechanism can quantitatıvely account for the experımental data on $k_{\mathrm{r}}$ at fields $\gtrsim 100 \mathrm{G}$.
\end{abstract}

\section{Introduction}

Magnetic-field dependent reaction yields and magnetic polarization phenomena arising from the $d y-$ namics of spin correlated radical parrs (RPs) have become a subject of major interest during the last twenty years (for reviews of. refs. [1-5]). The understanding of such phenomena requires the dynamics of spin motion as governed by various intra- and inter-radical type interactions, as well as the diffusional dynamics to be included into the description of chemical kinetics. A particular situation is encountered if RPs are generated in micellar supercages [6] where they may be trapped for time periods up to several microseconds during which their degree of spin correlation may be monitored through geminate recombination kinetics [7-19]

Normally RPs will be capable of undergoing some fast recombination process leading to a diamagnetic ground state product. Due to the spin conservation rule such a reaction will occur preferentially during radical pair encounters with singlet spin correlation (cf. below. however, for some invalidation of this spin conservation rule). RPs that have been created with triplet spin correlation usually have to undergo a triplet-to-singlet transition before they can recombine. Isotropic hyperfine coupling is a major mechanism for such spin transitions which in zero and low magnetic fields occur as coherent processes within a time span typically in the range of up to some ten nanoseconds. If the magnetic field strength exceeds the value of the characteristic hyperfine coupling, coherent $T_{ \pm} / S$ transitıons are suppressed and the $T_{ \pm}$substates have to exchange energy with the bath by incoherent $T_{1}$ processes in order to couple to the reactive singlet spin state.

Since $T_{1}$ processes in organic radicals occur in the time domain of typically fractions of $\mu$ s to several $\mu \mathrm{s}$, they are in the same order of magnitude as the cage time wherein spin correlation of micellar RPs matters. Therefore the $T_{1}$-type relaxation processes are borne out in the magnetic-field dependence of intramicellar recombination kinetics. This fact was first pointed out by Hayashi and Nagakura [19] who provided quantitative estimates for the contributions of varous relaxation mechanisms. It has been also evidenced by other workers [10-14]. As pointed out above, the micellar cage time of a RP, i.e. the time period during which radicals diffuse within the micelle, thereby undergoing a series of reencounters, de- 
termines to what extent spin relaxation processes can contribute to the recombination process. Therefore, experiments with a systematic variation of the cage time as obtained through a micellar size variation should provide particularly valuable information for assessing the dominant mechanisms operatıng in micellar RP spin relaxation.

Experiments of such type have been performed in this laboratory [15] utulizing RPs generated with initial triplet spin correlation by photoelectron transfer between aniline and thionine triplet molecules solubilized in microemulsion nanodroplets of variable size [18]. The radius of the water pool enclosed by a monolayer of CDBA (cetyldimethylbenzyl ammonium chloride) surfactant molecules in benzene as a bulk solvent was varied between 15 and 60 A. Size variation of normal micelles by using different chain lengths of surfactant molecules [16] or by varying the ionic strength [17] has also been reported. So far, however, our microemulsion data represent the most detalled quantitative study of the dependence of radical pair recombination kinetics on supercage size. In this paper we will provide a quanutative analysis of the role of spin relaxation in the magnetic-field dependent recombination kinetics. The cage-size dependence of RP spin relaxation following from this analysis prompted us to develop a more realistic theoretical treatment than previously available for spin relaxation caused by intermolecular electron-spin dipolar interaction in micelles.

\section{Determining rate parameters of spin relaxation from magnetokinetic data}

In a magnetic field the chemical kinetics of a RP diffusing within a micellar supercage may be represented by scheme $1[10,11,19]$. It uses first-order rate processes to describe spinselective recombination of RPS in the singlet spin state $\left(k_{s}\right)$ or in the triplet spin state $\left(k_{\mathrm{T}}\right)$, non-spinselective RP decay by escape of one radical from the micelle $\left(k_{\text {esc }}\right)$ and, furthermore, the spin transitions $\mathrm{T}_{0} \rightleftharpoons \mathrm{S}\left(k_{\mathrm{r}, 0}\right), \mathrm{T}_{ \pm} \vec{\rightleftharpoons} \mathrm{T}_{0}\left(k_{\mathrm{r}, 1}\right)$, and $\mathrm{T}_{ \pm} \rightleftharpoons \mathrm{S}\left(k_{r, 1}^{r}\right)$. Actually, coherent spin transitions as being due to isotropic hyperfine coupling (hfe) between the spin substates cannot be exactly described by stochastic rate processes. However, in fields several times higher than the characteristic hfc field in

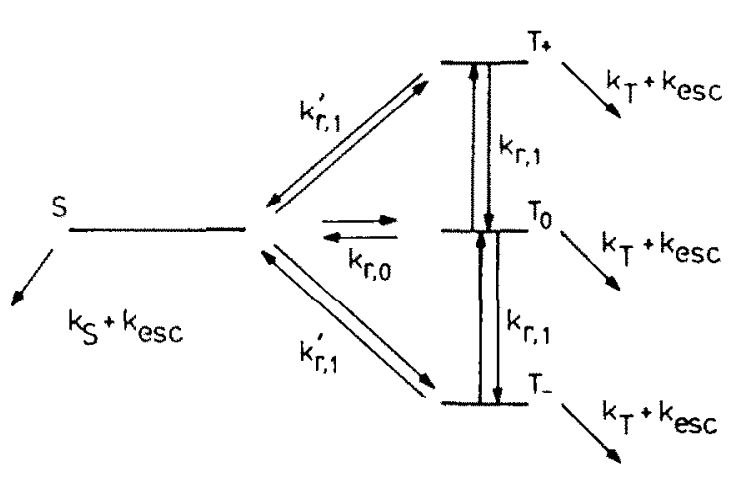

Scheme I.

the RP and on a time scale much longer than the inverse of the characteristic hfc frequency, as typical for intramicellar RP kinetics, such a global kinetic description can be justified. In this case $S$ and $T_{0}$ may be considered in equilibrium (i.e. the precise value of $k_{\mathrm{r}}$ is irrelevant), whereas $\mathrm{T}_{ \pm} \rightleftharpoons \mathrm{S}$ and $\mathrm{T}_{ \pm} \rightleftharpoons \mathrm{T}_{0}$ are purely incoherent $T_{1}$ processes. It is the magnetic-field dependence of these processes which is borne out in magnetokinetic behaviour at higher ( $\gg B_{\mathrm{hcc}}$ ) fields. Unless the RP energy is higher than that of the lowest locally excited triplet state, a situation encountered in some radical ion pairs [20] or in the primary event of photosynthesis [21], only recombination to some diamagnetic ground state product will be feasible. Spin conservation in this chemical reaction step makes it selective for the RP singlet spin state and $k_{\mathrm{s}}$ may approach the diffusion controlled limit. Recombination to diamagnetic products should be spin-forbidden for RPs encountering in a triplet spin state. Nevertheless such processes may occur through the effect of intermolecular spin-orbit coupling. For micellar RPs it was first demonstrated by Levin and Kuzmin [11] that one has to allow for such processes in order to account for the observation of magneticfield independent reaction channels open to $T_{+}$and $\mathrm{T}_{\text {- }}$ even in very high fields. For biradicals, evidence for such spin-inverted $k_{\mathrm{T}}$ processes was provided by Turro and coworkers $[13,14]$. The importance of the $k_{\mathrm{T}}$ processes should increase in the presence of heavyatom substituents, whereby the magnetic-field sensitivity of the overall kinetics should be decreased. Such heavy atom effects have been in fact observed [10,11]. In our previous work we suggested heavyatom enhanced spin-rotational relaxation, a mag- 
netic-field independent $T_{1}$ process as an explanation [10]. Recent experiments in this laboratory [22] with reverse micelles of variable size, however, have provided clear evidence that the heavy-atom induced quenching of the magnetic-field effect described in ref. [10] is largely due to a heavy-atom enhanced $k_{\mathrm{T}}$ process.

According to scheme 1 and the assumptions set out above, RPs generated in the triplet spin state will decay in a biexponential process with rate constants [11]

$k_{\mathrm{ots}(1.2)}=k_{\mathrm{csc}}+\frac{1}{4}\left(k_{\mathrm{S}}+3 k_{\mathrm{T}}\right)+k_{\mathrm{r}}+D_{1.2}$,

with

$D_{1.2}= \pm \sqrt{k_{\mathrm{r}}^{2}+\frac{1}{1 \mathrm{~h}}\left(k_{\mathrm{S}}-k_{\mathrm{T}}\right)^{2}}$

and

$k_{\mathrm{r}}=k_{\mathrm{r}, 1}+k_{\mathrm{r}, 1}^{\prime}$

A magnetic-field dependence of $k_{\mathrm{obs}}$ arises from the magnetic-field dependence of $k_{\mathrm{r}}(B)$. In the limit of low fields, where $k_{\mathrm{r}} \gg k_{\mathrm{s}}+k_{\text {ess }}$, the decay is monoexponential and

$k_{\mathrm{obs}}=k_{\mathrm{ohs} 2}=k_{\mathrm{esc}}+\frac{1}{4}\left(k_{\mathrm{S}}+3 k_{\mathrm{T}}\right)$.

In the limit of high fields $k_{\mathrm{r}} \ll k_{\mathrm{T}}$ and

$k_{\mathrm{obs}, 1}=k_{\mathrm{csc}}+\frac{1}{2}\left(k_{\mathrm{S}}+k_{\mathrm{T}}\right)$.

$k_{\mathrm{ohs.2}}=k_{\mathrm{esc}}+k_{\mathrm{T}}$.

Experimental information on the function $k_{\mathrm{r}}(B)$ can be obtained from analysis of $k_{\text {obs }}(B)$ utilizing the lowfield and high-field limiting expressions to fix $k_{\mathrm{S}}$ and $k_{\mathrm{T}}$. As mentioned in the introduction, in our previous experimental investigation [15] we obtained very detailed results on the magnetic-field dependent recombination kinetics of RPs in microemulsion nanodroplets of variable size. In that work a rate constant $k_{\text {rec }}(B)$ was evaluated which, in terms of the present notation, corresponds to

$k_{\mathrm{rec}}(B)=k_{\mathrm{obs} .2}(B)-k_{\mathrm{esc}}$.

The experimental data points for $k_{\mathrm{rec}}(B)$ for various values of $w$ are shown, e.g., in fig. 1 . The quantity $w$ is the molar ratio of water to surfactant. The radius of the water nanodroplet is expected to increase linearly with $w$ if the packing density of surfactant head groups on the surface of the water droplet is constant
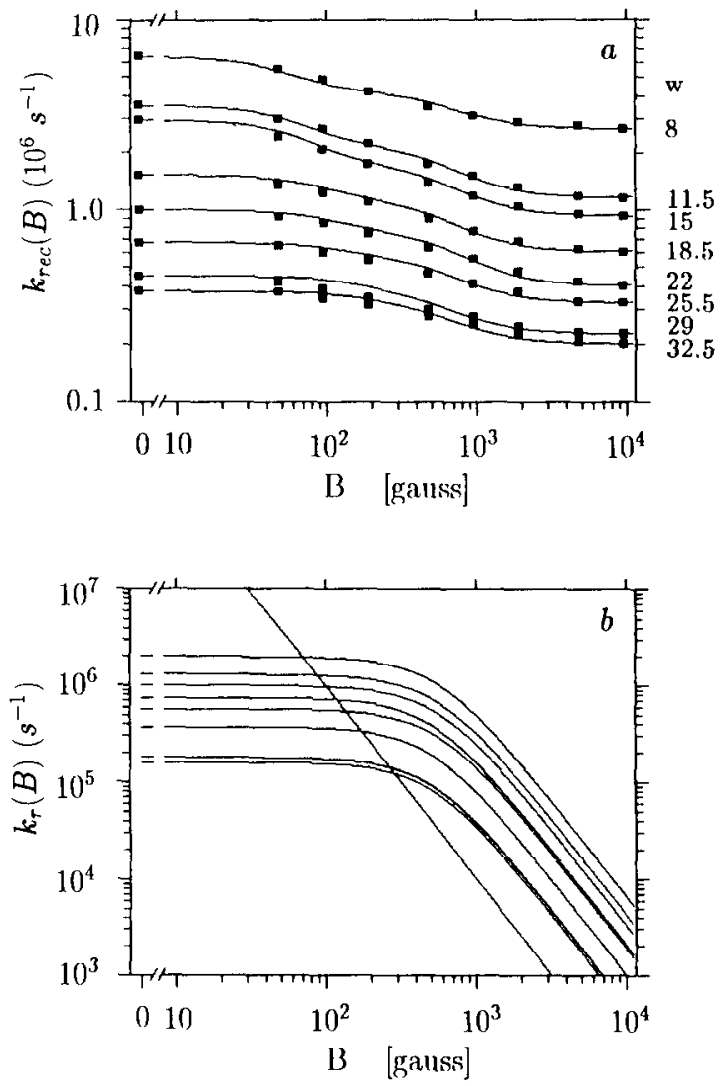

Fig. 1. (a) Data points: experımental results from ref. $[15]$ on the magnetic-field dependence of intramicellar recombination rate constant $k_{\text {Iec }}$ of thionıne/aniline triplet RPs in reverse micelles of variable water pool size characterized by parameter $w$ ( cf. text for definition ). Lines: theoretical fits using eqs. (1)-(7) and (8). (b) Graphical representation of the two contributions to $k_{\mathrm{r}}(B)$ used in (a). The straight line representung the $A_{1}$ term in eq. (8) is independent of $w$, the curves representing the $A_{2}$ term in eq. (8) correspond to increasing $w$ in the order from top to bottom

which seems to apply fairly well to typical water-inoil microemulsion systems [23]. With $k_{\mathrm{S}}$ and $k_{\mathrm{T}}$ fixed by the zero-field and high-field limit of $k_{\text {rcc }}$, the values of $k_{\mathrm{r}}(B)$ at intermediate fields $B$ can be uniquely determined from the experimental value of $k_{\text {rec }}(B)$ by using eqs. (1) and (2). Of course the experimental error in $k_{\text {rec }}(B)$ will lead to large uncertainties of $k_{\mathrm{r}}(B)$ as $k_{\mathrm{rec}}(B)$ approaches the high-field or low-field limit. Reliable values of $k_{\mathrm{r}}(B)$ to withın $\pm 30 \%$ may be obtained, however, in the field region between 100 and $2000 \mathrm{G}$. Here we found it convenient to describe 
the observed $k_{\mathrm{r}}(B)$ variation by an empirical expression of the form

$\hat{i}_{\Gamma}(B)=\frac{A_{1}}{\omega^{2}}+\frac{A_{2}}{1+\omega^{2} \tau_{\mathrm{c}}^{2}}$.

Here $\omega$ is the Larmor frequency and $A_{1}, A_{2}$ and $\tau_{\mathrm{c}}$ are adjustable parameters. The Lorentzian-type second term has the form of a spectral density of a stochastic perturbation with an exponential autocorrelation function of time constant $\tau_{\mathrm{c}}$. In this interpretation the quantity $A_{2}$ would be proportional to $\overline{\left|H_{ \pm .0}^{\prime}\right|^{2}} \tau_{\mathrm{c}}$, where $\left|H_{ \pm, 0}^{\prime}\right|^{2}$ is the mean square of the perturbation matrix element subject to stochastic modulation. The Lorentzian term was used to fit the highfield part of the field dependence of $k_{\text {rec }}(B)$ (cf. fig. 1). It turned out, then, that at low fields the saturation value $k_{1 c c}$ obtained on extrapolating the high-field fit, was too low to account for the observed limiting value in zero field. Actually, this is not surprising since we know that as $B \rightarrow 0$ the coherent $\mathrm{T}_{ \pm} \rightleftharpoons \mathrm{S}$ process must overtake the $T_{1}$ relaxation processes. So the first term in eq. (8) was introduced as an empirical expression to correct. in a formal way, for the contribution of cohcrent $T_{ \pm} / \mathrm{S}$ mixing at low ficlds. A constant value of the parameter $A_{1}\left(3 \times 10^{24} \mathrm{rad}^{2} \mathrm{~s}^{-3}\right)$ was used for all values of $u$. For $\tau_{c}$, too, a $w$-independent value $\left(10^{-10} \mathrm{~s}\right)$ could be used. The w-dependence of $k_{\text {reu }}(B)$ was fairly well reproduced by a $w$ dependent variation of $A_{2}$ only.

The results obtained for $k_{\mathrm{rec}}(B)$ by this empirical fitting procedure for $k_{\mathrm{r}}(B)$ are shown in fig. la together with the experimental data points. It should be noted that. in terms of Weller's theoretical $B_{1 / 2}^{\text {hf }}$ value [24], the hfc strength characteristic of the RPs considered here, is $30 \mathrm{G}$ [25] corresponding to an angular frequency of about $5 \times 10^{8} \mathrm{rad} \mathrm{s}^{-1}$, whereas the fastest recombination rate is less than $10^{7} \mathrm{~s}^{-1}$. So at fields $\gtrsim 100 \mathrm{G}$ the conditions for treating spin-dependent RP kinetics on the basis of scheme 1 should be well justified. The graphical representation of the two terms adding to $k_{\mathrm{r}}(B)$ according to eq. (8) is shown in fig. $1 \mathrm{~b}$. While for $w \leqslant 18.5$ there is some unrealistic inflexion behaviour in the calculated $k_{\mathrm{r}}(B)$ at a field of about $150 \mathrm{G}$ which is due to the crudeness of approximation in modelling the low-field effect by a general $A_{1} / \omega^{2}$ term, the relaxation behavlour in high fields is well reproduced by the second term in eq. (8) which may therefore be used as an empirical basis to discuss the dependence of the relevant spin relaxation mechanism on the radius of the intramicellar water pool.

The main conclusion from fig. $1 \mathrm{~b}$ is that $A_{2}$ depends very strongly on $w$. In fact. this dependence closely parallels the $w$-dependence of $k_{\text {rec }}(0)$ which is determined by the frequency of reencounters of the $\mathrm{RP}$ in the micellar supercage, an assumption based on the approximate $r_{\mathrm{M}}{ }^{3}$ dependence observed for $k_{\text {rec }}(0)$ [15]. where $r_{\mathrm{M}}$ denotes the radius of the intramicellar water pool. Such a behaviour of $A_{2}$ and hence of $\overline{\left|H_{ \pm 0}^{\prime}\right|^{2}}$ can only arise if the responsible perturbation is mainly due to an inter-radical type interaction. The principal mechanism to be considered in this regard is electron-spin dipolar interaction.

\section{Theory of RP spin relaxation due to electron-spin dipolar interaction in micellar cages}

\subsection{General formallsm}

Following the usual approach in the framework of first-order time-dependent perturbation theory ( $\mathrm{cf}$. ref. [26]) the transition probability per time unit between eigenstates $i$ and $j$ of the time-independent Hamiltonian is given by

$k_{l, i}(\omega)=k_{,, l}(\omega)=\hbar^{-2} \int_{-\infty}^{+\infty} G_{i l}(\tau) \mathrm{e}^{(\omega) \tau} \mathrm{d} \tau$.

where

$G_{i j}(\tau)=\left\langle H_{i l}^{\prime *}(t+\tau) H_{\iota j}^{\prime}(t)\right\rangle$

is the autocorrelation function of the perturbation matrix element $H_{\prime \prime}^{\prime}$, modulated by the fluctuations of some stochastic variables and having an average value of zero. In the case of dipolar interaction between the two electron spins in a RP $H^{\prime}$ is given by [26]

$H^{\prime}=\gamma_{\mathrm{c}}^{2} \hbar^{2}\left(\frac{S_{1} \cdot S_{2}}{r_{12}^{3}}-\frac{3\left(S_{1} \cdot r_{12}\right)\left(S_{2} \cdot r_{12}\right)}{r_{12}^{5}}\right)$.

It depends on the radius vector $\boldsymbol{r}_{12}$ between the two spins $S_{1}$ and $S_{2}$ and the relative orientations of the two spins with respect to each other and to the radius vector, respectively. These geometric parameters represent the stochatic variables that are modulated 
through the diffusional motion of the two radicals. Since $H^{\prime}$ commutes with $\left(S_{1}+S_{2}\right)^{2}$, the total spin of the RP cannot be changed by the effect of $H^{\prime}$, i.e. $H_{\mathrm{TS}}^{\prime}=0$ and hence $k_{\mathrm{r}, 1}^{\prime}=0$. The matrix elements required for $k_{r .1}$ are [26]

$$
\begin{aligned}
& H_{\mathrm{T}+\mathrm{T}_{0}}^{\prime}=-H_{\mathrm{T}-\mathrm{T}_{0}}^{\prime *} \\
& \quad=-\gamma_{\mathrm{c}}^{2} \hbar^{2} \frac{3}{4} \sqrt{2} r_{12}^{-3} \sin \theta \cos \theta \mathrm{e}^{-10},
\end{aligned}
$$

where $\theta$ and $\phi$ are polar and azimuthal angles, respectively, describing the orientation of the vector $\boldsymbol{r}_{12}$ in a Cartesian frame with the $z$-axis parallel to the axis of quantization defining the triplet substates $T_{+}, T_{0}$ and $\mathrm{T}_{-}$.

For convenience, we will define the correlation function

$$
\begin{gathered}
K_{\mathrm{T}_{ \pm} \mathrm{T}_{0}}(\tau) \equiv \frac{8}{9} \gamma_{\mathrm{e}}^{-4} \hbar^{-4} G_{\mathrm{T}_{ \pm} \mathrm{T}_{0}}(\tau) \\
=\left\langle\left(r_{12}^{-3} \sin \theta \cos \theta \mathrm{e}^{1 \phi}\right)_{t+\tau}\right. \\
\left.\left(r_{12}^{-3} \sin \theta \cos \theta \mathrm{e}^{-1 \phi}\right)_{t}\right\rangle
\end{gathered}
$$

and the spectral density function

$S_{\mathrm{T}_{ \pm} \mathrm{T}_{0}}(\omega)=\int_{-\varkappa^{\prime}}^{+\infty} K_{\mathrm{T}_{ \pm} \mathrm{T}_{0}}(\tau) \mathrm{e}^{\mid(\omega) \tau} \mathrm{d} \tau$

by means of which $k_{\mathrm{r}, 1}(B)$ can be represented as

$k_{1}(B)=\frac{9}{8} \gamma_{c}^{4} \hbar^{2} S_{\mathrm{T}_{ \pm} \mathrm{T}(\gamma)}\left(\gamma_{c} B\right)$.

\subsection{Previous treatment of dipolar interaction in micellar RPS}

Previous estimates of dipolar interaction between electron spins in micellar RPs usually refer to the paper by Hayashi and Nagakura [19] who used an expression implying a spectral density function of the form

$$
S_{\mathrm{T}_{ \pm \mathrm{T}_{0}}}(\omega)=\begin{array}{cc}
4 & r_{12}^{-6} \tau_{\mathrm{c}} \\
15 & 1+\omega^{2} \tau_{\mathrm{c}}^{2}
\end{array}
$$

Such a result corresponds to a situation where two spins are held at a fixed distance $r_{12}$ and the orientation of the radius vector undergoes isotropic rotational diffusion, characterized by a correlation time $\tau_{\mathrm{c}}$. It is realistically applicable to the case where the two spins are fixed within a rigid molecule undergoing rotational diffusion. However, application of eq.
(16) to a pair of radicals undergoing independent translational diffusion in a micelle can at best provide a suitable functional dependence on $\omega$ (cf. section 2) whereas an interpretation of the values to be used for $r_{12}$ and $\tau_{r}$ in terms of the basic physical parameters of the system is not straightforward. A realistic model of the process should provide the dependence of $k_{\mathrm{r} .1}$ on the parameters $r_{\mathrm{M}}, r_{0} . D_{1}, D_{2}$, i.e. the radius of the spherical volume accessible to the two radicals, the distance of their closest approach and their translational diffusion coefficients, respectively. In suggesting a dependence on these parameters only it is implicitly assumed that the point dipolar approximation may be applied in locating the electron spins and that the two radicals may be approximated as spherical particles with the spins localized in their centers, so that individual rotational motion of the two radicals does not cause a modulation of $H^{\prime}$. These assumptions represent, of course, approxımations in the case of aromatic $\pi$ radicals.

\subsection{Spin-dipolar relaxation by translational diffusion: adapting results for homogeneous solutions to RPs in micelles}

A general theory of magnetic relaxation due to spindipolar interaction between particles undergoing isotropic translational diffusion in homogeneous solutions has been developed by Torrey [27]. He obtained spectral densities for the timc-corrclation

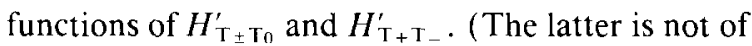
interest from the viewpoint of scheme 1 , since we will not investigate the case of differential population or depopulation of $T_{ \pm}$that might arise only in connection with a pronounced effect of the triplet mechanism (cf. ref. [2] ).) The diffusional model used by Torrey implies discrete jumps of spins with a Gaussian distribution of jump widths, characterized by an average mean square displacement $\left\langle r^{2}\right\rangle$ and distributed in time according to a Poisson distribution characterized by a time constant $\tau$. The relation with the diffusion constant $D$ of individual spins is

$\left\langle r^{2}\right\rangle=6 D \tau$.

For a spin interacting with other spins, independently diffusing in a homogeneous solution with an average concentration of $N$ (cf. fig. 2), Torrey de- 


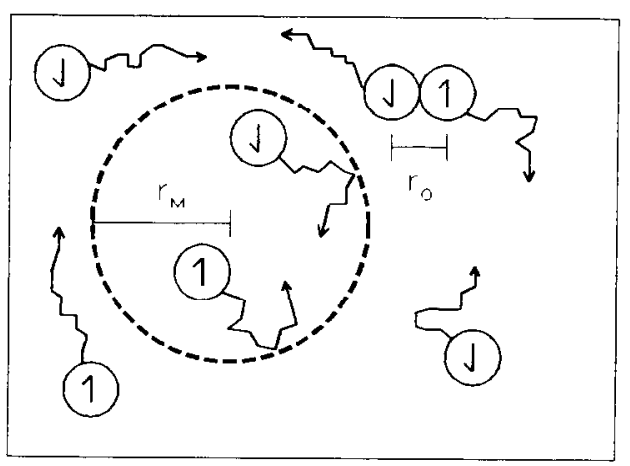

Fig. 2. Comparison of the situations corresponding to randomly diffusing radicals in homogeneous solutions and a RP diffusing withın a spherical micellar supercage.

rived the following expression for the spectral density $S_{\mathrm{T}_{ \pm} \mathrm{T}_{0}}(\omega)$ of the perturbation $H_{\mathrm{T}_{ \pm} \mathrm{T}_{0}}^{\prime}$ :

$S_{\mathrm{T}_{ \pm} \mathrm{T}_{0}}(\omega)=\frac{8 \pi}{15} N r_{0}^{-3} \omega^{-1} f(\alpha, x)$

with

$\alpha=\left\langle r^{2}\right\rangle /\left(12 r_{0}^{2}\right), \quad x=\sqrt{\omega r_{0}^{2} / D}$,

and

$$
\begin{aligned}
& f(\alpha, x)=\left(\frac{2}{x^{2}}\right)\left\{v\left(1-\frac{1}{u^{2}+v^{2}}\right)\right. \\
& +\left[u\left(1+\frac{1}{u^{2}+v^{2}}\right)+2\right] \mathrm{e}^{-2 u^{\prime}} \cos 2 u \\
& \left.+u\left(1-\frac{1}{u^{2}+v^{2}}\right) \mathrm{e}^{-2 u} \sin 2 u\right\},
\end{aligned}
$$

whereby

$$
\begin{aligned}
& u=\frac{1}{2} \sqrt{\alpha^{-1} q(1-q)}, \\
& v=\frac{1}{2} \sqrt{\alpha^{-1} q(1+q)}, \\
& q=\alpha x^{2} / \sqrt{1+\alpha^{2} x^{4}} .
\end{aligned}
$$

The spectral density $S_{\mathrm{T}_{ \pm \mathrm{T}}}(\omega)$ is proportional to the concentration of interactıng spins and can be considered as an uncorrelated superposition of all possible pair interactions of a particular spin with any other spin in the solution. Due to the $r_{12}^{-6}$ dependence of $K_{\mathrm{T} \pm \mathrm{T}_{0}}$. contributions to $S_{\mathrm{T} \pm \mathrm{T}_{0}}$ arise only from those parts of a pair's diffusion trajectory where $r_{12}$ ranges within several times $r_{0}$. the distance of closest ap- proach. Therefore, if the radicals are far apart from each other for most of time, $S_{\mathrm{T}_{ \pm} \mathrm{T}_{0}}$ is proportional to the frequency of pair collisions or of close encounters. If the "encounter volume" wherein appreciable contributions to $S_{\mathrm{T}+\mathrm{T}_{0}}$ arise, is small as compared to the volume accessible to a RP enclosed in a micellar supercage, there is no principle difference between the contribution of single encounters to spin-dipolar relaxation in homogeneous solution and in micelles and we may adapt eq. (18) to the casc of just onc RP in a micelle by substituting the inverse of the micellar volume

$I_{\mathrm{M}}^{r}=\frac{4}{3} \pi r_{\mathrm{M}}^{3}$

for $N$, yielding

$S_{\mathrm{T} \pm \mathrm{T}_{0}}(\omega)=\frac{2}{5} r_{\mathrm{M}}^{-3} r_{0}^{-3} \omega^{-1} f(\alpha, x)$.

\subsection{Monte Carlo treatment of spin-dipolar relaxation of $R P S$ by $3 D$ and $2 D$ diffusion in micelles}

In the treatment suggested in the last section, micellar boundary effects restricting diffusion during a RP encounter are neglected. In order to be able to judge the significance of such effects for situations of experimental interest and to account for other modes of diffusional restraints (in particular the motion of a RP on a spherical interface) we set up a computer program to calculate the perturbation matrix element $H_{\mathrm{T}_{ \pm} \mathrm{T}_{n}}^{\prime}(t)$ over individual diffusional trajectories chosen by a Monte Carlo method, and to obtain the corresponding correlation function $K_{T_{ \pm} T_{0}}(\tau)$ by averaging over a sufficiently long period of time.

To obtain the diffusional trajectories. random jumps were calculated for each radical at discrete time intervals $\Delta t$. The diffusional step widths into each direction were chosen to conform to a Gaussian probability distribution characterized, in one dimension, by a mean square of

$$
\left\langle r^{2}\right\rangle_{1 \mathrm{D}}=2 D \Delta t \text {. }
$$

To maintain an interradical separation wider than $r_{n}$, the distance of closest approach, a specular boundary condition for single radical jumps was applied. In treating $3 \mathrm{D}$ diffusion within the spherical boundary of the micellar aggregate also a specular boundary condition was applied, when a jump virtually would go to a position outside the sphere. In the case of $2 \mathrm{D}$ 
diffusion on a spherical interface the 2D random jump was first calculated to occur on a plane tangential to the actual position of the radical considered. Then, the position on the plane reached by the jump was radially projected onto the sphere.

The correlation function $K_{\mathrm{T}_{ \pm} \mathrm{T}_{0}}(\tau)$ was calculated for discrete values of $\tau$

$\tau_{n}=n \Delta \tau$,

with $n=0,1,2, \ldots, n_{\text {max }}$. Typically, the parameter values were $\Delta \tau=10 \mathrm{ps}, n_{\max }=200$. The computer calculation was organized in such a way that an array of $n_{\max } \Delta \tau / \Delta t$ values of $H_{T_{ \pm} T_{0}}^{\prime}\left(t_{i}\right)$ was kept in the memory and with increasing $t_{t}=i \Delta t$ the new values were entered, replacing the earlıest ones in a cyclic order. The averaging of $K_{\mathrm{T}+\mathrm{T}_{1}}\left(\tau_{n}\right)$ was typically extended over a time period of $0.5 \mathrm{~ms}$ during which about 5000 encounters of a RP took place. The Monte Carlo program was written in C language and was run on a Micro Vax 3600 . The time consumption for calculating $n=200$ values of $K_{\mathrm{T} \pm \mathrm{T} v}\left(\tau_{n}\right)$, each averaged over $5 \times 10^{7}$ diffusional steps, was about 33 hours (CPU). The degree of accuracy reached by the Monte Carlo calculation may be judged from comparison of the value obtained for $K_{\mathrm{T}_{ \pm} \mathrm{T}_{0}}(0)$ with the exact analytical result which can be derived as the mean square of the perturbation matrix element for a random distribution of RP constellations in the volume, or on the surface, of a sphere of radius $r_{M}$. Averaging over the angular coordinates yields

$K_{T_{ \pm} T_{0}}(0)=\frac{2}{15}\left\langle r_{12}^{-6}\right\rangle$.

Averaging over the distance $r_{12}$ yields in the $3 \mathrm{D}$ case:

$$
\begin{aligned}
& K_{\mathrm{T} \pm \mathrm{T}_{0}}(0)=r_{\mathrm{M}}^{-6} \frac{5+32 \rho_{0}^{-3}-36 \rho_{0}^{-2}+6 \ln \left(2 \rho_{0}^{-1}\right)}{\frac{15}{2}\left(32-32 \rho_{0}^{3}+18 \rho_{0}^{4}-\rho_{0}^{6}\right)} \\
& \simeq \frac{2}{15} r_{0}^{-3} r_{\mathrm{M}}{ }^{3} .
\end{aligned}
$$

and in the 2D case:

$$
\begin{aligned}
& K_{\mathrm{T}_{\perp} \mathrm{T}_{1}}(0)=r_{\mathrm{M}}^{-6} \frac{1}{15\left(4-\rho_{0}^{2}\right)}\left(\frac{1}{\rho_{0}^{4}}-\frac{1}{16}\right) \\
& \simeq \frac{1}{60} r_{0}^{-4} r_{\overline{\mathrm{M}}}^{-2},
\end{aligned}
$$

where $\rho_{0}=r_{0} / r_{M}$ and the approximations given apply to the limit $r_{\mathrm{M}} / r_{\mathrm{U}} \gg 1$.

Examples of results for $K_{\mathrm{T}_{ \pm} \mathrm{T}_{0}}(0)$ as obtained from a Monte Carlo calculation in comparison with analytically obtained values of $K_{\mathrm{T}_{ \pm} \mathrm{T}_{0}}(0)$ are given in table 1.

An example of a correlation function $K_{\mathrm{T} \pm \mathrm{T}_{0}}(\tau) \mathrm{ob}-$ tained from the Monte Carlo calculation is shown in fig. 3. Our numerical results could be fitted quite well by a sum of two exponentials

$K_{\mathrm{T}_{ \pm} \mathrm{T}_{0}}(\tau)=K_{\mathrm{T}_{ \pm} \mathrm{T}_{0}}(0)\left(a_{1} \mathrm{e}^{-\tau / \tau_{1}}+a_{2} \mathrm{e}^{-\tau / \tau_{2}}\right)$,

with $u_{1}+u_{2}=1$,

Characteristic results demonstrating the dependence of the values $a_{1}, \tau_{1}$ and $\tau_{2}$ on the radius $r_{\mathrm{M}}$ of the micellar sphere are listed in table 2 . From these data one may conclude that, at least in the region of interest in relation with our experiments $\left(r_{\mathrm{M}} \geqslant 20 \AA\right)$. $a_{1}, \tau_{1}$ and $\tau_{2}$ are rather insensitive to $r_{\mathrm{M}}$. Therefore, in all our applications for the final modelling of the magnetokinetic data, we used the following average sets of these parameters:

Table 1

Comparison of $K_{\mathrm{T} \pm \mathrm{T}_{0}}(0)$ values (1n units of $10^{-7} \AA^{-0}$ ) obtained by Monte Carlo calculations a and by analytical formulae (26) and (27)

\begin{tabular}{lllll}
\hline$r_{\mathrm{M}}(\dot{4})$ & 20 & 25 & 35 & 50 \\
\hline $\begin{array}{l}\text { 3D Monte Carlo } \\
\text { eq. (26) }\end{array}$ & 2.080 & 1.097 & 0.4248 & 0.1646 \\
& 2.045 & 1101 & 0.4245 & 0.1518 \\
2D Monte Carlo & 1.659 & 1.065 & 0.5448 & 0.2645 \\
eq. (27) & 1.644 & 1.048 & 0.5332 & 0.2608 \\
\hline
\end{tabular}

a) $r_{0}=4 \AA, D=5 \times 10^{-7} \mathrm{~cm}^{2} \mathrm{~s}^{-1}, n_{\max }=5 \times 10^{7}$.

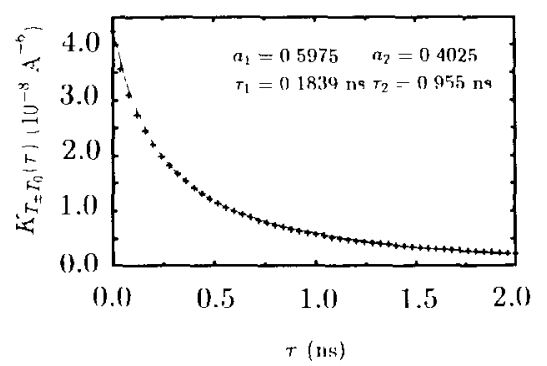

Fig. 3. Correlation function $K_{\mathrm{T}_{ \pm} \mathrm{T}_{0}}(\tau)$. The data points represent values obtained by a Monte Carlo calculation using $D=5 \times 10^{-7}$ $\mathrm{cm}^{2} \mathrm{~s}^{-1}, r_{0}=4 \AA, r_{M}=35 \AA$. The line corresponds to the best fit of a biexponential decay (cf. eq. (28) and table 2 ). 
Table 2

Parameters $\tau_{1}, \tau_{2}$ and $a_{1}$ determined by Monte Carlo calculation for a RP in micelles of different radi1 $r_{M}$. The values of other parameters were $D_{1}=D_{2}=5 \times 10^{-7} \mathrm{~cm}^{2} \mathrm{~s}^{-1}$ and $r_{0}=4 \AA$

\begin{tabular}{lllll}
\hline Dim & $r_{\mathrm{M}}(\AA)$ & $\tau_{1}(\mathrm{~ns})$ & $\tau_{2}(\mathrm{~ns})$ & $a_{1}$ \\
\hline $3 \mathrm{D}$ & 20 & 0.1789 & 0.903 & 0.5760 \\
& 25 & 0.2035 & 1.063 & 0.6171 \\
& 35 & 0.1839 & 0955 & 0.5975 \\
& 50 & 01644 & 0.846 & 05395 \\
$2 \mathrm{D}$ & 20 & 0.2200 & 1.502 & 0.5000 \\
& 25 & 0.2242 & 1.629 & 0.5150 \\
& 35 & 0.2098 & 1.485 & 0.4826 \\
& 50 & 0.2210 & 1.557 & 0.4944 \\
\hline
\end{tabular}

3D case:

$$
a_{1}=0.60, \quad \tau_{1,0}=0.19 \mathrm{~ns}, \quad \tau_{\urcorner, 0}=0.97 \mathrm{~ns},
$$

2D case:

$$
a_{1}=0.50, \quad \tau_{1,0}=0.22 \mathrm{~ns}, \quad \tau_{2,0}=1.54 \mathrm{~ns} .
$$

The $\tau$ values given refer to a value of $D_{0}=5 \times 10^{-7}$ $\mathrm{cm}^{2} \mathrm{~s}^{-1}$ for the diffusion constants of both radicals. As long as we are only interested in a time resolution well beyond $\Delta t$, a change in $D$ will only correspond to a linear rescaling of the time axis. Thus the values of $\tau_{1,0}$, and $\tau_{2,0}$ obtained when employing the standard value $D_{0}$, must be changed to $\tau_{1(2), 0} D_{0} / D$ for a general value $D$ of the diffusion constant.

Using the parameter $K_{\mathrm{T}+\mathrm{T}_{0}}(0)$, obtained analytically, and the parameters $a_{1}, \tau_{1}$, and $\tau_{2}$ obtained by the Monte Carlo calculation, the following expression for the spectral density can be calculated:

$S_{\mathrm{MC}, \mathrm{T}_{ \pm} \mathrm{T}_{0}}(\omega)=2 K_{\mathrm{T}_{ \pm} \mathrm{T}_{0}}(0)\left(\frac{a_{1} \tau_{1}}{1+\omega^{2} \tau_{1}^{2}}+\frac{a_{2} \tau_{2}}{1+\omega^{2} \tau_{2}^{2}}\right)$.

By analogy with eq. (23) a function $f_{\mathrm{MC}}(\alpha, x)$ can be defined as

$f_{\mathrm{MC}}(\alpha, x) \equiv \frac{5}{2} r_{\mathrm{M}}^{3} r_{0}^{3} \omega S_{\mathrm{MC}, \mathrm{T}_{ \pm} \mathrm{T}_{0}}(\omega)$.

which may be compared to Torrey's result for $f(\alpha$, $x)$. For $r_{\mathrm{M}}=25 \AA$ this comparison of $f(\alpha, x)$ with $f_{\mathrm{Mc}}(\alpha, x)$ is shown in fig. 4 . As can be seen, both functions are in fair agreement up to $\log \left(x^{2}\right) \approx 1.8$ corresponding to $\omega \approx 2 \times 10^{10} \mathrm{rad} \mathrm{s}^{-1}$. The discrepancy at higher frequencies is due to the $10 \mathrm{ps}$ time step employed in the Monte Carlo calculation. Actually, to fit the Torrey function over the whole fre-

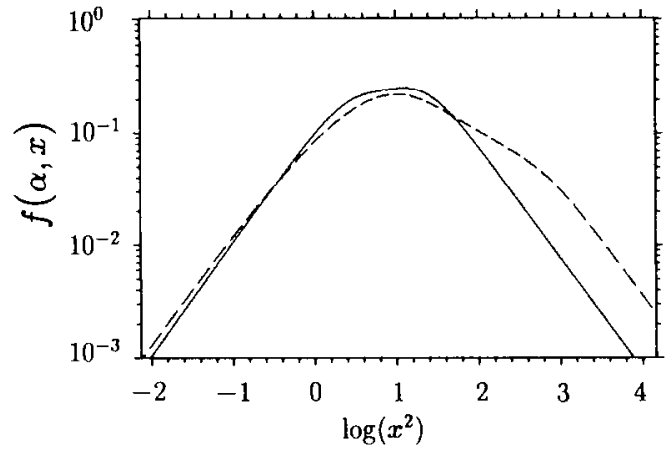

Fig. 4. Comparison of the function $f(\alpha, x)$ as obtained from eq. (20) (dashed line) and from eq (30) involving a Monte Carlo calculation (solid line). The parameter values are given in the text.

quency range, at least three correlation times would be required. Anyhow, for the purpose of our application the high-frcquency region need not be reproduced too exactly, since at high fields the rate constant of spin relaxation drops below the value of $k_{\mathrm{T}}$ determining the high-field limit of $k_{\text {rec }}$.

In fig. 5 the actual field dependence of the rate constant $k_{\mathrm{r} .1}$ as obtained by eq. (15) on the basis of the Monte Carlo calculation of $S_{\mathrm{MC} . \mathrm{T} \pm \mathrm{T}_{0}}(\omega)$ is shown for a series of micellar radii in the $3 \mathrm{D}$ and $2 \mathrm{D}$ diffusional case. It is clear that the general behaviour of $k_{\mathrm{r}, 1}(B)$ as obtained from the analysis of the magnetokinetic data (fig. 1b), is borne out by the theoretical curves. In the next section it will be shown that, by choosing physically consistent sets of the parameters determining RP diffusion, the experimental results for $k_{\text {rec }}(B)$ can be well reproduced.

\section{Fitting the parameters of the spin-dipolar relaxation mechanism to the experimental $\boldsymbol{k}_{\mathrm{rec}}(B)$}

In our model treatment, spin-dipolar relaxation in the RP is determined by the four parameters $r_{\mathrm{M}}, r_{0}$, $D_{1}$ and $D_{2}$. In application of this model to simulate the observed magnetic-field dependence of $k_{\text {rec }}$ we adopted a reasonable value of $r_{0}=4 \AA$ and used the same value of $D_{1}=D_{2}=D$ for both radicals of the pair. The remaining parameters, $D$ and $r_{\mathrm{M}}$, were considered as adjustable for each value of $w$, the experimental parameter fixing the size and other properties of the microemulsion droplet. In order to compare the 

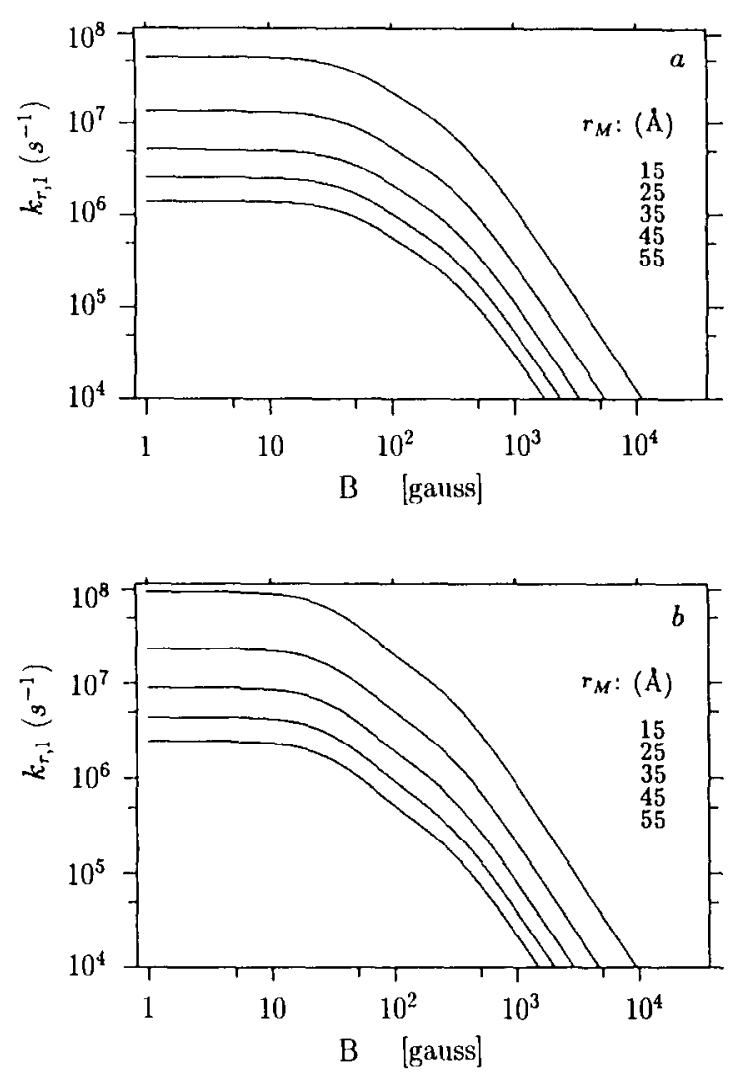

Fig. 5. Magnetic-field dependence of $k_{r}$, calculated for various supercage radii $r_{M}$ by eq. (15) and using the Monte Carlo method to obtain $S_{\mathrm{T}_{ \pm} \mathrm{T}_{0}}\left(\gamma_{\mathrm{e}} B\right)$ Other parameters are $D=5 \times 10^{-7} \mathrm{~cm}^{2} \mathrm{~s}^{-1}$. $r_{u}=4 \AA$. (a) Case of $3 D$ diffusion withın a sphere, (b) case of 2D diffusion on the surface of a sphere.

theoretical to the experimental results we calculated $k_{\text {rec }}(B)$ using eqs. (1-4) and (7). The values of $k_{\mathrm{S}}$ and $k_{\mathrm{T}}$ were obtained from the zero-field and highfield limit of the experimental $k_{\mathrm{rec}}(B)$ according to eqs. (4) and (6), and $k_{\mathrm{r}, 1}(B)$ was calculated according to the modified Torrey formula (23) or using the results of the Montc Carlo method (cq. (29)). No effect of isotropic hyperfine coupling on $\mathrm{T}_{ \pm} \rightleftharpoons \mathrm{T}_{0}, \mathrm{~S}$ was taken into account. Therefore the obtained $k_{\text {rec }}(B)$ will be inevitably too low in the field region below $\approx 50 \mathrm{G}$ and this field region must not be considered in judging the agreement between the theoretical and the experimental result.

In fig. 6 is shown how, for a fixed value of $D$, a variation of $r_{M}$ affects the theoretical field depen-

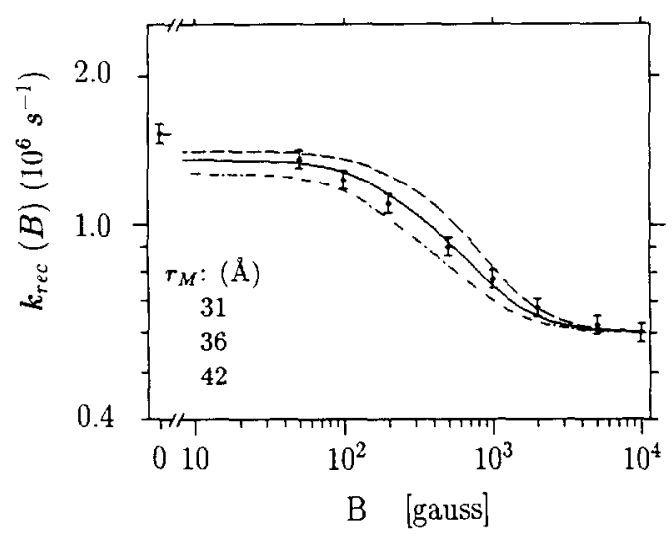

Fig. 6. Demonstration of a fit of the experimental $k_{\text {rec }}(B)$ (case $w=18.5$ ) by a suitable choice of parameter $r_{\mathrm{M}}$. The calculation of $k_{\text {rec }}(B)$ was based on eqs. (1)-(4), (6), (7), (15), (26) and (29), using $k_{\mathrm{s}}=4.3 \times 10^{6} \mathrm{~s}^{-1}, k_{\mathrm{T}}=6 \times 10^{5} \mathrm{~s}^{-1}, D=1 \times 10^{-6} \mathrm{~cm}^{2}$ $\mathrm{s}^{-1}$ and $r_{0}=4 \AA$.

dence of $k_{\text {rec }}(B)$. It is demonstrated that, through the fit, $r_{\mathrm{M}}$ can be fixed with a relative accuracy of about $\pm 15 \%$. In the following we varied the value of $D$ and determined the best-fit value of $r_{\mathrm{M}}$ for every $w$. In fig. 7 representative results are shown for calculations pertinent to $3 \mathrm{D}$ diffusion. There is no significant difference between the results from applying the modified Torrey formula or the Monte Carlo method. As can be seen in fig. $7 \mathrm{c}$, for a value of $D=5 \times 10^{-7} \mathrm{~cm}^{2}$ $\mathrm{s}^{-1}$ the drop of $k_{\mathrm{rcc}}(B)$ is too pronounced. For $D=1 \times 10^{-6} \mathrm{~cm}^{2} \mathrm{~s}^{-1}$ (fig. $7 \mathrm{~b}$ ) the fit is very good for the full field range $>50 \mathrm{G}$ and for all values of $w$, whereas for $D=2 \times 10^{-6} \mathrm{~cm}^{2} \mathrm{~s}^{-1}$ (fig. 7a) $k_{\text {rec }}(B)$ is too low at fields $\leqslant 100 \mathrm{G}$. Considering that this discrepancy would perhaps be less when taking the effect of hyperfine coupling properly into account, the best-fit value of $D$ may be assumed at about 1$2 \times 10^{-6} \mathrm{~cm}^{2} \mathrm{~s}^{-1}$. It should be noted that the series of $r_{\mathrm{M}}$ values, assigned to the experimental values of $w$ exclusively by means of the fitting procedure, is rather insensitive to the chosen value of $D$ once this is in the vicinity of its best-fit value.

A fitting procedure as the one documented for the 3D diffusion model in fig. 7 was also applied using the $2 \mathrm{D}$ diffusion model. The quality of the fit that may be obtained with the latter is similar to that achieved with the 3D diffusion model. The parameter values obtained for $D$ and $r_{\mathrm{M}}(w)$ from best fits 

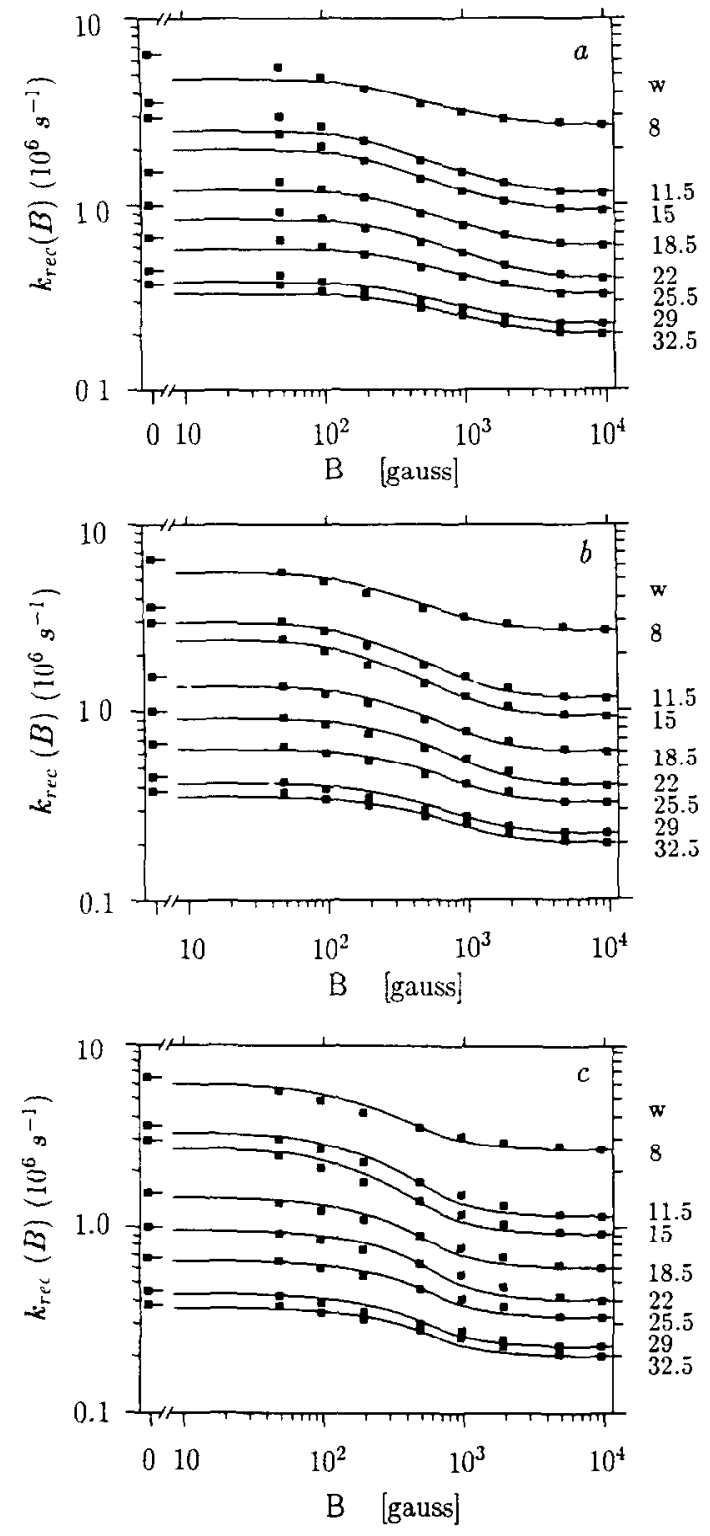

Fig. 7 Best fits obtained in varying parameter $D$ and $r_{\mathrm{M}}$ to model the observed $k_{\text {rec }}(B)$ for vanous $w$ by calculations using eqs. (1)(4), (6), (7), (15), (26) and (29). With increasing order of $w$ values the best fits were obtained usıng $r_{M}=25,29,32,36,38$, $45,54,56 \AA$, independent of $D$. The $D$ values are constant in each figure with the values (a) $D=2 \times 10^{-6} \mathrm{~cm}^{2} \mathrm{~s}^{-1}$, (b) $D=1 \times 10^{-6}$ $\mathrm{cm}^{2} \mathrm{~s}^{-1}$. (c) $D=05 \times 10^{-6} \mathrm{~cm}^{2} \mathrm{~s}^{-1}$. with either of the two models are represented in fig. 9.

\section{Discussion}

In section 3 we adapted Torrey's results for spinrelaxation, as induced by dipolar interaction between freely diffusing spins in homogeneous solution, to the situation of one RP in a micellar cage. In doing so, we pointed out that Torrey's relation can be conceived as a sum of individual spin-pair encounters. Here we note that a similar view is adopted when deriving the (first-order) rate constant of diffusioncontrolled recombination of a RP in the confined space of micelle. In homogeneous solution with molecular concentration $N=N_{\mathrm{A}} c$ of radicals the pseudo first-order rate constant for radical disappearance by recombination is

$k_{\text {diff }}^{(1)}=4 \pi r_{0} D^{\prime} N$

with $D^{\prime}=D_{1}+D_{2}$, the sum of diffusion constants of the reacting particles. Applying eq. (31) to the case of two radicals in a micelle of volume $\frac{4}{3} \pi r_{\mathrm{M}}^{3}$ the effective concentration $N$ of the second reactant is just the inverse of this volume and

$k_{\mathrm{d} \text { fff }}^{\mathrm{mic}}=3 D^{\prime} r_{0} r_{\mathrm{M}}^{-3}$.

Eqs. (31) and (32) are the analogues to eqs. (18) and (23) in section 3.3. For a rigorous derivation of eq. (32) by solving the diffusion equation for a pair of reactants with appropriate boundary conditions $\mathrm{cf}$. refs. [28] and [29]. Carrying the analogy between eqs. (23) and (32) further, one could write

$k_{\mathrm{r}, 1}(\omega)=p_{\mathrm{r}}(\omega) k_{\mathrm{d} \text { iff }}^{11}$.

defining a field-dependent relaxation probability per encounter $p_{\mathrm{r}}(\omega)$. For RPs in micellar cages this may be expressed as

$p_{\mathrm{r}}(\omega) \equiv k_{\mathrm{r}, 1} / k_{\mathrm{d} \text { Iff }}^{\mathrm{m} 1 \mathrm{f}}=\frac{3}{8} \gamma_{\mathrm{e}}^{4} \hbar^{2} \frac{r_{\mathrm{M}}^{3}}{r_{0} D^{\prime}} S_{\mathrm{T}_{ \pm} \mathrm{T}_{0}}(\omega)$.

With $S_{\mathrm{T}_{ \pm T_{0}}}(\omega)$ as given in eq. (29) and using the two-exponential approximation of eq. (26) for $K_{\mathrm{T}_{ \pm} \mathrm{T}_{0}}(0)$, the zero-field value $p_{\mathrm{r}}(0)$ takes the form

$p_{\mathrm{r}}(0)=\frac{1}{20} \gamma_{\mathrm{e}}^{4} \hbar^{2} \frac{a_{1} \tau_{1,0} D_{0}+a_{2} \tau_{2,0} D_{0}}{r_{0}^{4} D^{2}}$. 
As stated above, for $r_{M} \gg r_{0}$ the expression $a_{1} \tau_{10} D_{0}+a_{2} \tau_{2,0} D_{0} \approx 3 \AA$ is independent of $D_{0}$ and the micellar radius. Using the latter value, the final result is

$p_{\mathrm{r}}(0) \approx 1.54 \times 10^{4} \frac{\AA^{8}}{\mathrm{~ns}^{2}} r_{0}^{-4} D^{-2}$.

The quantity $p_{\mathrm{r}}(\omega)$ may be used to assess the validity of the perturbation treatment for $k_{\mathrm{r}, 1}$. To warrant this validity, one should have

$p_{r}((1))<1$.

With $r_{0}=4 \AA$ and $D=10^{6} \mathrm{~cm}^{2} \mathrm{~s}^{-1}$ as typically applied in our simulations we obtain $p_{\mathrm{r}}(0)=0.6$, indicatıng that condition (37) is met at zero field and, a fortiori, in the field range where the relaxation becomes magnetokinetically relevant.

With the theoretical calculation of $k_{r, 1}$ it has been possible in section 4 to simulate the observed $k_{\mathrm{rec}}(B)$ curves for a range of $w$ values. Thereby the diffusion constants and the micellar radii were assigned bestfit values. the physical significance of which will now be discussed.

Let us first address the results obtained for $r_{\mathrm{M}}$ as a function of $w$. Independent experimental information on the relation of these quantities is available from the literature. Miller [30] determined aggregation numbers of CDBA in water/benzene microemulsions using excimer formation of pyrene sulphonate as a probe, while Jada et al. [31 ] applied the method of fluorescence quenching of ruthenium tris (bipyridyl) by methyl viologen to obtain the same kind of formation with chlorobenzene as the oil component. From the aggregation number $g$ and the parameter $w$ the radius of the water pool $r_{\mathrm{wp}}$ is obtained as [31]

$r_{\mathrm{wp}}=\left[\frac{3 g\left(w^{\prime} l^{\prime} \mathrm{w}+l^{\prime} \mathrm{C}^{-}\right)}{4 \pi}\right]^{1 / 3}$.

where $v_{\mathrm{W}}$ and $v_{\mathrm{Cl}}$ - are the molecular volumes of water and $\mathrm{Cl}^{-}$ion, respectively. The results of Miller and of Jada et al. are shown in fig. 8. Both sets of data may be well represented by a single straight line described by

$r_{\mathrm{up}}=1.61 w+4.28$.

For hydrophilic radicals as employed in our experi-

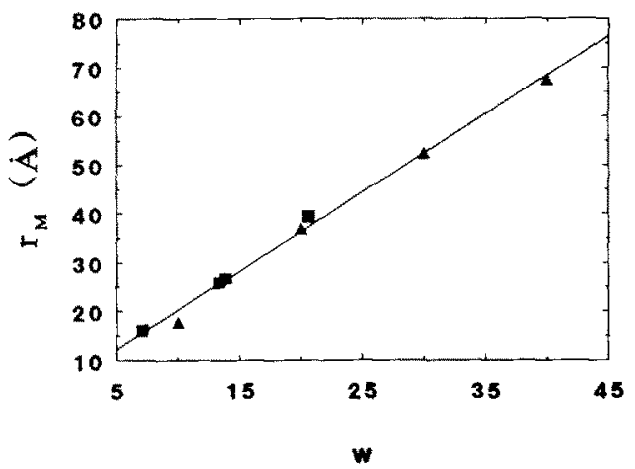

Fig. 8. Literature results on the dependence of water pool radius $r_{M}$ on parameter $w$. ( $\mathbf{m}$ ) Data from Miller [30] for microemulsions of $\mathrm{CDBA} / \mathrm{H}_{2} \mathrm{O}$ in benzene. ( $\mathbf{A}$ ) Data from Jada et al. [31] for microemulsions of $\mathrm{CDBA} / \mathrm{H}_{2} \mathrm{O}$ in chlorobenzene
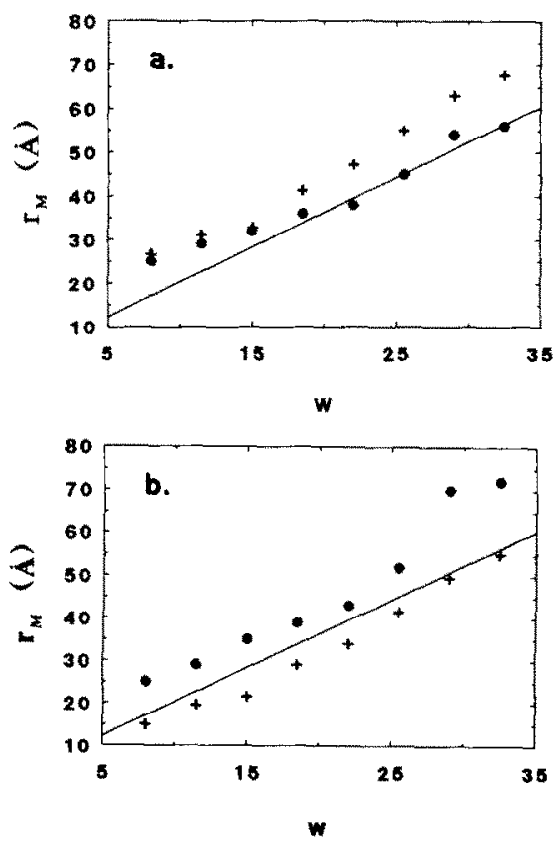

Fig. 9. Dependence of water pool radius $r_{M}$ on parameter $w$ as evaluated in this work: (O) from the magnetic-field dependence of $k_{\mathrm{rec}}(B)$ (cf. fig. 7 ) and ( + ) from assigning $k_{\mathrm{s}}$ to the diffusioncontrolled limit (cf. text). (a) For 3D, (b) For 2D diffusion of the KP in the volume or on the surface of the supercage represented by the intramicellar water pool. The values of the radical diffusion constants were (a) $D=1 \times 10^{-6} \mathrm{~cm}^{2} \mathrm{~s}^{-1}$, (b) $D=1.5 \times 10^{-6} \mathrm{~cm}^{2} \mathrm{~s}^{-1}$. The straight line corresponds to the best fit of the literature data as displayed in fig. 8 . 
ments one would expect $r_{\mathrm{M}}=r_{\mathrm{wp}}$. In fig. 9a, $\mathrm{b}$ we compare the $r_{M}\left(w^{\prime}\right)$ data from the relaxational analysis of $k_{\text {Icc }}$ with the literature results. For both $3 \mathrm{D}$ and $2 \mathrm{D}$ diffusional models the relaxational results are quite compatible with the literature data. Although the data from the $3 \mathrm{D}$ model may compare somewhat more favourably, we do not consider this difference as significant.

From the zero-field and high-field limit of $k_{\text {rec }}$ determıned in our experiments we could obtain values of the rate constant $k_{\mathrm{S}}$ pertaining to spin-allowed intramicellar backward electron transfer in the RP. This process may be considered as diffusion-controlled and hence $k_{\mathrm{s}}$ provides another access to the diffusional parameters of the RP. For diffusion-controlled recombination in a spherical volume of radius $r_{M}$ the following expression was obtained by Hatlec ct al. [28]

$k_{\text {diff }}^{3 \mathrm{D}}=\frac{3 D^{\prime} r_{0} / r_{\mathrm{M}}}{r_{\mathrm{M}}^{2}\left(1-r_{0} / r_{\mathrm{M}}\right)^{2}}$,

which, in the limit $r_{\mathrm{M}} / r_{0} \gg 1$, adopts the form of eq. (32). If we assume that $k_{\mathrm{S}}=k_{\mathrm{d} \text { iff }}^{3 \mathrm{D}}$, eq. (40) can be used to obtain an effective value of $r_{\mathrm{M}}$ from $k_{\mathrm{s}}$. In fig. 9a we show the corresponding values of $r_{M}\left(w^{\prime}\right)$ obtained from eq. (40) when using the same values of $D^{\prime}$ and $r_{0}$ as for modelling the $w$ dependence of $k_{\mathrm{r} .1}$. The agreement with the other data on $r_{M}$ is satisfactory.

For the case of 2D diffusion on a spherical surface no closed-form analytical expression for $k$ diff is available. Some numerical results obtained by a series expansion are given in ref. [28]. We used these to verify solutions obtained in our laboratory by numerical integration of the 2D diffusion equation on a computer. Using the latter method we then determined $r_{\mathrm{M}}$ values that would yield the experimental $k_{\mathrm{S}}$ values with the $2 \mathrm{D}$ diffusional model when employing the same values of $r_{0}$ and $D^{\prime}$ as in the previous methods. The results of $r_{M}$ versus $w$ for this case are also shown in fig. $9 \mathrm{~b}$. In fact, these values come very close to the $r_{\mathrm{M}}(w)$ line representing the data from refs. [30] and [31].

In concluding the discussion of the $r_{\mathrm{M}}\left(w^{\prime}\right)$ results we note that, within the error margin to be reasonably expected for our model calculation, both $2 \mathrm{D}$ and $3 \mathrm{D}$ diffusional model yield reasonable results and neither possibility can be ruled out on this basis.
However, we also have to consider the values obtained for the diffusion constants $\left(D^{\prime} \approx 2 \times 10^{-6} \mathrm{~cm}^{2}\right.$ $\mathrm{s}^{-1}$ according to the $3 \mathrm{D}$ model. $D^{\prime} \approx 3 \times 10^{-6} \mathrm{~cm}^{2} \mathrm{~s}^{-1}$ according to the $2 \mathrm{D}$ model). They should be compared to a value of about $1 \times 10^{-5} \mathrm{~cm}^{2} \mathrm{~s}^{-1}$ [32] for the radicals in neat water and they indicate an about ten-fold increased viscosity experienced by the radicals in the polar interior of the microemulsion droplets as compared to bulk water. We do not know of microviscosity determinations inside the reverse micellar aggregates of the present system with the cationic CDBA. Corresponding results are available for water-in-oil microemulsions with the anionic AOT in aliphatic solvents like isooctane [33]. Those results indicate an appreciable dependence of the effective viscosity (i) on the size of the water pool, and (ii) on the location of the water. Thus, between $r_{M}=20$ and $50 \AA$, the effective viscosity is reported [33] to drop from about $90 \mathrm{cP}$ to about $70 \mathrm{cP}$ in the water layer adjoining the head-group zone, and from about $15 \mathrm{cP}$ to about $5 \mathrm{cP}$ in the interior of the water pool. The effective viscosity of $\approx 10 \mathrm{cP}$ corresponding to our $D^{\prime}$ value is intermediate to the latter bounds and would suggest that the reactants move rather freely through the interior of the water pool. It must be emphasized, however, that in contrast to what has been reported on the AOT system, our results do not indicate a $w$-depcndence of the effective viscosity. Furthermore, we want to point out that experiments in our laboratory [34] with the same type of RP. however donor and acceptor held in the interface region by the anchoring effect of long aliphatic substituents, indicate a behaviour very similar to that for the RP studied in this paper. The latter observation argues in favor of $2 \mathrm{D}$ diffusional motion of the RP. In any case, more detailed studies on the diffusional behaviour and the microviscosity within the CDBA reverse micellar aggregates seem to be desirable.

Finally. we turn to the question of the contribution of RP spin relaxation mechanisms other than electron-spin dipolar interaction. For organic $\pi$ radicals without heavy atoms but with appreciable hyperfine coupling the anisotropy of the latter is usually the main source of stochastic perturbation leading to spin relaxation. Magnetic isotope effects $\left({ }^{1} \mathrm{H} /{ }^{2} \mathrm{H}\right.$ and ${ }^{12} \mathrm{C} /$ ${ }^{13} \mathrm{C}$ ) observed in the field region of several hundred to several thousand gauss $[12,35,36]$ provide evi- 
dence that hyperfine coupling must be involved in electron spin relaxation of RPs, too.

For a $R P$, the $T_{1}$ relaxation induced in one radical by the anisotropic hfc with one nuclear spin ( $I=$ $1 / 2$ ) makes a contribution to $k_{\mathrm{r}}$ given by

$\Delta_{\mathrm{hfc}} k_{\mathrm{r}}(\omega)=\frac{2^{2}}{12} \frac{(t: t) \tau_{\mathrm{c}}}{1+\omega^{2} \tau_{\mathrm{c}}^{2}}$,

where $\tau_{c}$ is the orientational correlation time of the radical experiencing the coupling and the anisotropy of the $h f c$ is defined as

$$
(t: t) \equiv \sum_{t}\left(a_{u}-\bar{a}\right)^{2} .
$$

In deriving eq. (41) the rate constants given in ref. [19] for the individual hyperfine transitions, occuring in the $\mathrm{hfc}$-active radical, have been combined. Although a more complicated expression would have to be used in the presence of several coupling nuclei on each radical we may draw a few conclusions from the simple expression of eq. (41).

Could the $w$-dependence of $k_{\mathrm{r}}(B)$, borne out in fig. 1 , be explained on the basis of eq. (41)? There, the only quantity that might depend on $w$ to an appreciable extent is $\tau_{\mathrm{c}}$. Combining Debye's expression for $\tau_{\mathrm{c}}$ with the Stokes-Einstein relation for the translational diffusion constant one can write [37]

$\tau_{\mathrm{c}, l}=\frac{2}{9} \frac{r_{t}^{2}}{D_{l}}$

where $r_{\text {, }}$ is the hydrodynamic radius of the radical $i$ and $D_{l}$ is its translational diffusion coefficient. As reported above, $D_{t}$ might increase with the radius of the water pool. In consequence, $\tau_{\mathrm{c}}$ would decrease and so would $\Delta_{\mathrm{hfc}} k_{\mathrm{r}}(0)$. In the field-dependent region, however, where $\omega^{2} \tau_{\mathrm{c}}^{2} \gg 1, \Delta_{\mathrm{hrc}} k_{\mathrm{r}}(0)$ would increase with increasing water pool size. This is clearly at variance with the observed behaviour. On the other hand, in the empirical analysis of $k_{\mathrm{rec}}(B)$ (cf. fig. 1) it was shown that the data are consistent with some contribution to $k_{\mathrm{r}}$ independent of $w$, and overriding the $w$ dependent contribution at fields below 100 to $200 \mathrm{G}$, depending on $w$. Actually, in this field region the coherent $T_{ \pm} / T_{0}, S$ mixing mechanism is expected to be completely negligible (note that Weller's theoretical $B_{1 / 2}^{\text {hff }}$ is only $30 \mathrm{G}$ in our system). Thus, there is room for a relaxational contribution of the anisotropic hy- perfine coupling mechanism. It might well account for the $A_{1} / \omega_{0}^{2}$ contribution in the empirical fit. Adopting this interpretation we can derive from the high-field limit of eq. (41):

$$
\lim _{(1) \gg \tau_{\mathrm{c}}^{-1}} \Delta_{\mathrm{hrc}} k_{\mathrm{r}}(\omega)=\frac{\gamma_{\mathrm{e}}^{\frac{2}{e}}}{12} \frac{(t: t)}{\left.\tau_{\mathrm{c}} \omega\right)^{2}}=\frac{A_{1}}{\omega^{2}} \text {. }
$$

Using $A_{1}=3 \times 10^{24} \mathrm{rad}^{2} \mathrm{~s}^{-3}$ (cf. section 2) and $\tau_{\mathrm{c}}=4 \times 10^{-10} \mathrm{~s}$, following from eq. (43) with values of $D_{t}=5 \times 10^{-7} \mathrm{~cm}^{2} \mathrm{~s}^{-1}$ and $r_{t}=3 \AA$, we obtain $(t: t)=47 \mathrm{G}^{2}$, a value that seems compatible with the order of magnitude of hyperfine coupling in the two radicals [25]. Of course, in a more quantitative treatment of this mechanism, eq. (41) would have to be more specifically adapted to the case of two nuclei with spin $I=1$, since the $N$-hyperfine coupling constants are the dominant ones in thionine and aniline radicals.

Finally, an experimental result shall be mentioned, which gives a clear indication of the role of anisotropic hyperfine coupling in systems of the kind investigated in this work. We measured the field dependence of $k_{\text {rec }}$ under variation of the electron donor through the series: (aniline), $m$-F-aniline, $o$-F-aniline. $p$-F-aniline [38]. The experimental $B_{1 / 2}$ values. defined as the field where $k_{\text {rec }}$ adopts the average valuc of the zero-field and the high-field ( $10 \mathrm{kG}$ ) limit, were determined as $(140), 125,160,580 \mathrm{G}$ (data for microemulsion with $w=15$ ). This series clearly correlates with the radical cation's electron-spin density at the F-substituent. Aromatic ${ }^{19} \mathrm{~F}$ substituents exhibit a stronger hyperfine coupling than ${ }^{1} \mathrm{H}$ atoms and, what is more important here, the anisotropy of the ${ }^{19} \mathrm{~F}$-coupling is much larger. Thus the isotropic hyperfine coupling constants in the para position of the aniline cation are $a\left({ }^{1} \mathrm{H}\right)=9.6 \quad \mathrm{G} \quad$ [39] and $a\left({ }^{19} \mathrm{~F}\right)=20.8 \mathrm{G}$ (the latter value was reported for dimethylaniline [39], in aniline it might be somewhat larger). From these the corresponding anisotropies can be estimated [26] as $(t: t)_{p-\mathrm{H}} \approx 50 \quad \mathrm{G}^{2}$ and $(t: t)_{p-\mathrm{F}} \approx 3750 \mathrm{G}^{2}$.

From eqs. (1), (2), (4) and (6) one can derive the following useful condition for the relaxation constant $k_{\mathrm{r}}$ at the field $B_{1 / 2}$ :

$k_{\mathrm{T}}\left(B_{1 / 2}\right)=\frac{3}{16}\left(k_{\mathrm{S}}-k_{\mathrm{T}}\right)$.

Using this equation, a value of $1.5 \times 10^{6} \mathrm{~s}^{-1}$ is obtained for $k_{\mathrm{r}}\left(B_{1 / 2}\right)$ under the conditions of the above- 
mentioned experiments. According to eq. (41) this value of $k_{\mathrm{r}}$ will be obtained with $p$-F-aniline when $\omega \approx 1.2 \times 10^{10}$ rad $\mathrm{s}^{-1}$, corresponding to a field of about $700 \mathrm{G}$. This value is in quite good agreement with the experimental $B_{1 / 2}$ value.

\section{Conclusions}

The theoretical treatments of electron-spin dipolar relaxation of RPs in micellar supercages allow precise assessment of the contribution of this mechanism in terms of parameters of immediate physical significance, viz. $r_{0}, r_{M}, D_{1}$ and $D_{2}$. Whether the electron-spin dipolar relaxation mechanism will be borne out in the magnetic-field dependence of RP recombination kinctics depends on whether its contribution to $k_{\mathrm{r}}$, particularly in the field region where $k_{r}(B) \approx \frac{3}{16}\left(k_{\mathrm{S}}-k_{\mathrm{T}}\right)$ (cf.eq. (45)). is the dominating one. This we have shown to be the case for RPs enclosed in the polar interior of the nanometer-sized droplets of water-in-oil microemulsions. Here the electron-spin dipolar relaxation mechanism dominates the magnetokinetic behaviour of RPs at fields $\gtrsim 100 \mathrm{G}$, yielding $B_{1 / 2}$ values between 140 and 400 G. The relaxational effect of anisotropic hyperfine coupling seems to govern the region between $50 \mathrm{G}$ and $100 \mathrm{G}$, whereas coherent T-S mixing takes over at still lower fields. For the microemulsion-type supercages considered here, $1 \mathrm{t}$ has been rationalized that the magnetokinetic $B_{1 / 2}$ value is determined by relaxation through anisotropic hyperfine interaction only with radicals of extremely high values of anisotropic hyperfine coupling ( $p$-F-aniline radical cation).

Finally, we want to point out that care has to be taken when judging the contribution of electron-spin dipolar relaxation from empirically determined values of $\overline{H_{\mathrm{T}_{ \pm} \mathrm{T}_{(\mathrm{S})}}^{\prime 2}}$, as has been done, e.g., in ref. [11]. Combining eqs. (10), (13) and (25) one has

$\overline{H_{\mathrm{T}_{ \pm} \mathrm{T}_{0}}^{\prime 2}}=\frac{3}{20} \gamma_{\mathrm{e}}^{4} \hbar^{4}\left\langle r_{12}^{-6}\right\rangle$.

Hence, from $\overline{H_{\mathrm{T}}^{\prime 2} \mathrm{r}_{0}}$ one can deduct a value for an effective radius of dipolar interaction, defined as

$r_{\mathrm{eff}}^{\mathrm{dd}} \equiv\left(\frac{20}{3} \frac{\overline{H_{\mathrm{T}}^{t_{\mathrm{T}}^{2} \mathrm{~T}_{0}}}}{\gamma_{\mathrm{e}}^{4} \hbar^{4}}\right)^{-1 / 6}$.

For deciding whether the observed $\overline{H_{\mathrm{T}_{ \pm} \mathrm{T}_{0}(\mathrm{~S})}^{\prime}}$ may be due to the electron-spin dipolar relaxation mecha- nism, this effective radius must not be compared to $r_{\mathrm{M}}$, but to the theoretical value of $\left\langle r_{12}^{-6}\right\rangle^{-1 / 6}$ given by (cf. cqs. (25) and (26))

$\left\langle r_{12}^{-6}\right\rangle^{-1 / 6}=\sqrt{r_{0} r_{M}}<r_{M}$.

Using, for example, $r_{0}=4 \AA$ and $r_{\mathrm{M}}=20 \AA$ one obtains $r_{\text {eff }}^{\mathrm{dd}} \approx 9 \AA$ which is considerably smaller than $r_{\mathrm{M}}$ and rather close to the value of $11-12 \AA$ derived from the empirically determined $\overline{H_{\hat{\mathrm{T}}+\mathrm{T}_{n}}^{2}}$ denoted $\mid V^{2}$ in ref. [11].

\section{Acknowledgement}

We gratefully acknowledge financial support of this work by the Deutsche Forschungsgemeinschaft and by the Fonds der Chemischen Industrie. JQW is also obliged to the Ministerium für Wissenschaft und Kunst des Landes Baden-Wïrtemberg for a one-year scholarship.

\section{References}

[1] K.M. Salıkhov, Yu.N. Molın, R.Z. Sagdeev and A.L. Buchachenko, Spin Polarization and Magnetic Effects in Radical Reactions (Elsevier, Amsterdam, 1984).

[2] U.E. Steiner and T. Ulnch, Chem. Rev. 89 (1989) 51.

[3] U.E. Steiner and H.-J Wolff, in: Photochemistry and Photophysics, Vol. 4, ed. J.J. Rabek (CRC Press, Boca Raton, 1991) p 1 .

[4] H. Hayashi, in: Photochemistry and Photophysics, Vol 1 , ed. J.J. Rabek (CRC Press, Boca Raton, 1990) p. 59.

[5] K.A. McLauchlan and U.E. Steiner, Mol. Phys. 73 (1991) 241.

[6] N.J. Turro and G C. Weed, J Am. Chem. Soc. 105 (1983) 1861.

[7] Y. Sakaguch and H. Hayash1. Chem. Phys. Letters 87 (1982) 539

[8] Y. Tanimoto, M. Takashima and M. Itoh, Bull. Chem. Soc. Japan 57 ( 1984 ) 1747.

[9] C. Evans, K.U. Ingold and J C. Sca1ano, J Phys. Chem. 92 (1988) 1257

[10] T. Ulrıch, U.E. Steiner and W. Schlenker. Tetrahedron 42 (1986) 6131 .

[11] N.J. Levin and V.A. Kuzmun. Chem. Phys. Letters 165 (1990) 302.

[12] N.J. Turro, M B. Zimmt and I.R. Gould, J. Phys. Chem. 92 (1988) 433.

[13] M.B. Zimmt, C. Doubleday Jr. I R. Gould and N.J. Turro, J. Am. Chem. Soc. 107 (1985) 4724.

[14] Ch. Doubleday Jr., N J. Turro and J.-F. Wang, Accounts Chem. Res. 22 (1989) 199. 
[15] T. Ulrich and U.E. Steiner, Chem. Phys. Letters 112 (1984) 365.

[16] P.P. Levin and V.A. Kuzmın, Bull. Acad. Sc1. USSR Div. Chem. Sci. 36 (1987) 691; 95 (1991) 1880.

[17] P.P. Levin and V.A. Kuzmin, Bull. Acad. Sci. USSR Div. Chem. Sci. 37 (1988) 224.

[18] U.E. Steıner, in: Structure and Reactivity in Reverse Micelles, ed. M.P. Pllen1 (Elsevier, Amsterdam, 1989) p. 156.

[19] H. Hayashi and N. Nagakura, Bull. Chem. Soc. Japan 57 (1984) 322.

[20] H.-J. Werner, IH. Staerk and A. Weller, J. Chem. Phys. 68 (1978) 2419; Chem. Phys. Letters 96 (1983) 24.

[21] S.G. Boxer, C.E.D. Chidsey and M.G. Roelofs, Annu. Rev. Phys. Chem. 34 (1983) 389.

[22] D. Baumann, Ph.D. Thesis, Universität Konstanz, in preparation.

[23] H.-F. Eicke and P. Kvita, in: Reverse Micelles, eds. P.L. Luis1 and B.E. Straub (Plenum Press, New York, 1984) p. 21.

[24] A. Weller. F. Nolting and H. Staerk, Chem. Phys. I etters 96 (1983) 24

[25] W. Schlenker, T. Ulnch and U.E. Steiner, Chem. Phys. Letters 103 (1983) 118.
[26] A. Carrington and A.D. McLachlan, Introduction to Magnetıc Resonance (Chapman and Hall, London, 1979).

[27] H.C. Torrey, Phys. Rev. 92 (1953) 962.

[28] M.D. Hatlee, J.J. Kozak, G. Rothenberger, P.P. Infelta and M. Grätzel, J. Phys. Chem. 84 (1980) 1508.

[29] U. Gösele, U.K.A. Kleın and M. Hauser, Chem. Phys. Letters 68 (1979) 291.

[30] D.J. Miller, Ph.D. Thesss, Universität Stuttgart (1977).

[31] A. Jada, I. I ang, R. Zana, R. Makhloufi, E. Hirsch and S.J. Candau, J. Phys. Chem. 94 (1990) 387.

[32] U.E. Steiner and W. Haas, J. Phys. Chem. 95 (1991) 1880.

[33] P.E. Zinsli, J. Phys. Chem. 83 (1979) 3223.

[34] W. Haas, Ph.D. Thesis, Unıversitat Konstanz (1991).

[35] N.J. Turro, M.-F. Chow, C.-J. Chung, G.C. Weed and B. Kráutler, J. Am. Chem. Soc 102 (1980) 4843.

[36] Y. Sakaguchı, H. Hayashi and S. Nagakura, J. Phys. Chem. 86 ( 1982 ) 3177.

[37] K. Lüders and K.M. Salikhov, Chem. Phys. 117 (1987) 113. [38] J.Q. Wu and U.E. Steiner, to be published.

[39] B.M. Latta and R.W Taft, I. Am. Chem. Soc. 89 (1967) 5172 . 